\title{
APLICAÇÃO DO ESTIMADOR "MAXIMUM LIKELIHOOD" EM UM TESTE DE VIDA SEQÜENCIAL COM UMA DISTRIBUIÇÃO DE AMOSTRAGEM WEIBULL DE TRÊS PARÂMETROS
}

\author{
Daniel I. De Souza Jr. ${ }^{1}$ \\ Daniele Rocha Fonseca ${ }^{2}$
}

\begin{abstract}
RESUMO: Um mecanismo de teste de vida seqüencial com uma distribuição Weibull de três parâmetros, no qual existe informações significativas relativas aos valores dos parâmetros de forma e de escala do modelo Weibull representando o produto sendo testado, já foi analisado anteriormente por De Souza (2003), De Souza (2004a,b). Nesse trabalho iremos desenvolver um mecanismo de teste de vida seqüencial no qual a distribuição de amostragem será o modelo Weibull de três parâmetros e o produto sendo analisado é um novo componente metalúrgico. Assumiremos que o valor da vida mínima ou parâmetro de localização será diferente de zero. Para estimarmos os parâmetros de forma, de escala e de vida mínima do modelo Weibull de três parâmetros utilizaremos o estimador "Maximum Likelihood" em uma situação de teste de vida truncado por falhas. O emprego do método do Maximum Likelihood para estimar os parâmetros do modelo Weibull de três parâmetros poderá apresentar problemas no que se refere às condições de regularidade ou aderência, veja Murthy et al., (2004), Blischke, (1974) e Zanakis and Kyparisis, (1986). Para resolvermos o problema de falta de "regularidade" ou "aderência" acima mencionada utilizaremos uma modificação proposta por Cohen, et al. (1984). Um exemplo irá ilustrar a aplicação do mecanismo de truncagem do teste de vida seqüencial proposto.
\end{abstract}

PALAVRAS-CHAVES: Teste de Vida Seqüencial, Teste de Hipóteses, Mecanismo de Truncagem, Estimador "Maximum Likelihood".

ABSTRACT: A sequential life testing approach with a three-parameter Weibull underlying sampling distribution in which there was significant information about the shape and scale parameters of the Weibull model representing the product under analysis was analyzed before by De Souza, 2003 [1], De Souza, 2004a,b [2], [3]. In this work, we will develop a sequential life testing approach in which the underlying sampling distribution is the three-parameter Weibull model and the product being analyzed is a new metallurgical component. We will be assuming that the location parameter or minimum life is different from zero. To estimate the shape, scale and minimum life of the threeparameter Weibull model we will use a maximum likelihood approach for censored failure data. The standard maximum likelihood method for estimating the parameters of the three parameter Weibull model can have problems since the regularity conditions are not met, see Murthy, et al., 2004 [4], Blischke, 1974 [5] and Zanakis and Kyparisis, 1986 [6]. To overcome the "no regularity" problem, we will apply a modification proposed by Cohen, et al., 1984 [7].We will also develop a truncation mechanism for the three-parameter Weibull distribution. An example will develop the proposed truncated sequential life testing approach for the three-parameter Weibull model.

KEY-WORDS: Sequential Life-Testing, Hypothesis Testing, Truncation Mechanism, Maximum Likelihood Estimator.

\footnotetext{
${ }^{1}$ Universidade Federal Fluminense, Programa de Doutorado em Eng. Civil, daniel.desouza@hotmail.com

${ }^{2}$ Universidade Estadual do Norte Fluminense, Laboratório de Produção.

danielerfonseca@yahoo.com.br
} 


\section{INTRODUÇÃO}

A distribuição Weibull de três parâmetros possui um parâmetro de vida mínima $\varphi$ o qual representa a vida mínima da característica ou componente sendo analisado, um parâmetro de forma $\beta$ o qual especifica a forma da distribuição, e um parâmetro de escala $\theta$ o qual representa a vida característica da distribuição. Todos esses três parâmetros são positivos. A função densidade da distribuição Weibull de três parâmetros é dada por:

$$
f(t)=\frac{\beta}{\theta}\left(\frac{t-\phi}{\theta}\right)^{\beta-1} \exp \left[-\left(\frac{t-\phi}{\theta}\right)^{\beta}\right] ; t \geq 0
$$

As situações de teste de hipóteses foram dadas por Kapur e Lamberson (1977), e também por De Souza (2004a).

1. Para o parâmetro de escala $\theta$ :

$\mathrm{H}_{0}: \theta \geq \theta_{0} ; \mathrm{H}_{1}: \theta<\theta_{0}$

A probabilidade de se aceitar $\mathrm{H}_{0}$ será dada por $(1-\alpha)$ se $\theta=\theta_{0}$. Agora, se $\theta$ $=\theta_{1}$, onde $\theta_{1}<\theta_{0}$, a probabilidade de se aceitar $\mathrm{H}_{0}$ será ajustada para o nível inferior $\gamma$.

2. Para o parâmetro de forma $\beta$ :

$\mathrm{H}_{0}: \beta \geq \beta_{0} ; \mathrm{H}_{1}: \beta<\beta_{0}$

A probabilidade de se aceitar $\mathrm{H}_{0}$ será dada por $(1-\alpha)$ se $\beta=\beta_{0}$. Caso $\beta=\beta_{1}$ onde $\beta_{1}<\beta_{0}$, a probabilidade de se aceitar $\mathrm{H}_{0}$ será também ajustada para o nível inferior $\gamma$.

3. Para a vida mínima $\varphi$ :

$\mathrm{H}_{0}: \varphi \geq \varphi_{0} ; \mathrm{H}_{1}: \varphi<\varphi_{0}$

Novamente, a probabilidade de se aceitar $\mathrm{H}_{0}$ será dada por $(1-\alpha)$ se $\varphi=\varphi_{0}$. Agora, no caso de $\varphi=\varphi_{1}$ onde $\varphi_{1}<\varphi_{0}$, a probabilidade de se aceitar $\mathrm{H}_{0}$ será também ajustada para o nível inferior $\gamma$.

\section{TESTE SEQÜENCIAL}

De acordo com Kapur e Lamberson (1977) e De Souza (2001), para o modelo Weibull de três parâmetros, a relação seqüencial probabilística (SPR) será dada por SPR $=\mathrm{L} 1,1,1, \mathrm{n} / \mathrm{L} 0,0,0, \mathrm{n}$.

De acordo com De Souza (2003), para o modelo Weibull de três parâmetros, teremos:

$$
\begin{gathered}
\operatorname{SPR}=\left(\frac{\beta_{1}}{\theta_{1}} \times \frac{\theta_{0}^{\beta_{0}}}{\beta_{0}}\right)^{\mathrm{n}} \prod_{i=1}^{\mathrm{n}}\left[\frac{\left(\mathrm{t}_{\mathrm{i}}-\phi_{1}\right)^{\beta_{1}-1}}{\left(\mathrm{t}_{\mathrm{i}}-\phi_{0}\right)^{\beta_{0}-1}}\right] \times \\
\exp \left[-\sum_{\mathrm{i}=1}^{\mathrm{n}}\left(\frac{\left(\mathrm{t}_{\mathrm{i}}-\phi_{1}\right)^{\beta_{1}}}{\theta_{1}^{\beta_{1}}}-\frac{\left(\mathrm{t}_{\mathrm{i}}-\phi_{0}\right)^{\beta_{0}}}{\theta_{0}^{\beta_{0}}}\right)\right]
\end{gathered}
$$

A região contínua será dada por $\mathrm{A}$ $<$ SPR $<\mathrm{B}$, onde $\mathrm{A}=\gamma /(1-\alpha) ; \mathrm{B}=(1-$ $\gamma) / \alpha$. Iremos aceitar a hipótese nula $\mathrm{H}_{0}$ se $\mathrm{SPR} \geq \mathrm{B}$ e iremos rejeitar $\mathrm{H}_{0}$ se $\mathrm{SPR} \leq \mathrm{A}$. Agora, caso $\mathrm{A}<\mathrm{SPR}<\mathrm{B}$, analisaremos mais uma observação. Então, teremos:

$$
\begin{gathered}
\frac{\gamma}{(1-\alpha)}<\left(\frac{\beta_{1}}{\beta_{1}} \times \frac{\theta_{0}^{\beta} \beta_{0}}{\beta_{0}}\right)^{\mathrm{n}} \prod_{\mathrm{i}=1}^{\mathrm{n}}\left[\frac{\left(\mathrm{t}_{\mathrm{i}}-\phi_{1}\right)^{\beta_{1}-1}}{\left(\mathrm{t}_{\mathrm{i}}-\phi_{0}\right)^{\beta_{0}-1}}\right] \\
\times \exp \left[-\sum_{\mathrm{i}=1}^{\mathrm{n}}\left(\frac{\left(\mathrm{t}_{\mathrm{i}}-\phi_{1}\right)^{\beta_{1}}}{\theta_{1}^{\beta_{1}}}-\frac{\left(\mathrm{t}_{\mathrm{i}}-\phi_{0}\right)^{\beta}}{\theta_{0}^{\beta_{0}}}\right)\right]< \\
<\frac{(1-\gamma)}{\alpha}
\end{gathered}
$$

Obtendo-se os logaritmos naturais de todos os termos da equação acima e após alguma manipulação algébrica, obteremos:

$$
\begin{aligned}
& \operatorname{nln}\left(\frac{\beta_{1}}{\beta_{1}} \times \frac{\theta_{0}^{\beta_{0}}}{\beta_{0}}\right)-\ln \left[\frac{(1-\gamma)}{\alpha}\right]<X< \\
& <\operatorname{nln}\left(\frac{\beta_{1}}{\left.\beta_{1}^{\beta_{1}} \times \frac{0}{\beta_{0}}\right)}+\ln \left[\frac{(1-\alpha)}{\gamma}\right](1)\right. \\
& X=\sum_{i=1}^{n}\left(\frac{\left(\mathrm{t}_{\mathrm{i}}-\phi_{1}\right)^{\beta}}{\theta_{1}^{\beta_{1}}}-\frac{\left(\mathrm{t}_{\mathrm{i}}-\phi_{0}\right)^{\beta}}{\theta_{0}^{\beta_{0}}}\right)-
\end{aligned}
$$




$$
\begin{aligned}
-\left(\beta_{1}-1\right) \times & \sum_{i=1}^{n} \ln \left(t_{i}-\phi_{1}\right)+\left(\beta_{0}-1\right) \times \\
& \times \sum_{i=1}^{n} \ln \left(t_{i}-\phi_{0}\right)
\end{aligned}
$$

3. TAMANHO ESPERADO DA AMOSTRA DO TESTE DE VIDA SEQÜENCIAL PARA EFEITO DE TRUNCAGEM

De acordo com Mood e Graybill (1963), uma expressão para o tamanho esperado da amostra do teste de vida seqüencial para efeito de truncagem $\mathrm{E}(\mathrm{n})$, será dada por:

$$
E(n)=\frac{P(\theta, \beta) \ln A+[1-P(\theta, \beta)] \ln B}{E(w)}
$$

De acordo com De Souza (2003), para o modelo Weibull de três parâmetros, teremos:

$$
\begin{gathered}
\mathrm{E}(\mathrm{w})=\ln \left(\frac{\beta_{1}}{\theta_{1}} \times \frac{\theta_{0}^{\beta_{0}}}{\beta_{0}}\right)+\left(\beta_{1}-1\right) \times \\
\times \mathrm{E}\left[\ln \left(\mathrm{t}-\phi_{1}\right)\right]-\left(\beta_{0}-1\right) \times \\
\times \mathrm{E}\left[\ln \left(\mathrm{t}-\phi_{0}\right)\right]-\frac{1}{\theta_{1}^{\beta_{1}}} \mathrm{E}\left[\left(\mathrm{t}-\phi_{1}\right) \beta^{\beta_{1}}\right]+ \\
+\frac{1}{\theta_{0}^{\beta_{0}}} \mathrm{E}\left[\left(\mathrm{t}-\phi_{0}\right)^{\beta_{0}}\right]
\end{gathered}
$$

Novamente, $\mathrm{A}=\gamma /(1-\alpha) ; \mathrm{B}=(1-$ $\gamma) / \alpha$. O Anexo (1) apresenta as soluções para cada uma das partes da equação (4).

\section{ESTIMADOR MAXIMUM} LIKELIHOOD PARA O MODELO WEIBULL DE TRÊS PARÂMETROS: TRUNCAGEM POR FALHAS

O estimador "maximum likelihood" para os parâmetros de forma, escala e vida mínima de uma distribuição de amostragem Weibull de três parâmetros, em uma situação de teste de vida truncado por falhas, será dado por:

$$
\mathrm{L}(\beta ; \theta ; \varphi)=\mathrm{k} !\left[\prod_{i=1}^{\mathrm{r}} \mathrm{f}\left(\mathrm{t}_{\mathrm{i}}\right)\right]\left[1-\mathrm{F}\left(\mathrm{t}_{\mathrm{r}}\right)\right]^{\mathrm{n}-\mathrm{r}}
$$

$\mathrm{L}(\beta ; \theta ; \varphi)=\mathrm{k} !\left[\prod_{\mathrm{i}=1}^{\mathrm{r}} \mathrm{f}\left(\mathrm{t}_{\mathrm{i}}\right)\right]\left[\mathrm{R}\left(\mathrm{t}_{\mathrm{r}}\right)\right]^{\mathrm{n}-\mathrm{r}} ; \mathrm{t}>0$

$\operatorname{Com~} f\left(t_{i}\right)=\frac{\beta}{\theta^{\beta}}\left(t_{i}-\varphi\right)^{\beta-1} e^{-\left(t_{i}-\varphi / \theta\right)}{ }^{\beta}$

e com $\mathrm{R}\left(\mathrm{t}_{\mathrm{r}}\right)=\mathrm{e}^{-\left(\mathrm{t}_{\mathrm{r}}-\varphi / \theta\right)^{\beta}}$, teremos:

$$
\begin{aligned}
& \mathrm{L}(\beta ; \theta ; \varphi)=\mathrm{k} ! \frac{\beta^{\mathrm{r}}}{\theta^{\beta r}}\left[\prod_{i=1}^{\mathrm{r}}\left(\mathrm{t}_{\mathrm{i}}-\varphi\right)\right]^{\beta-1}- \\
& \mathrm{e}^{-\sum_{\mathrm{i}=1}^{\mathrm{r}}\left(\mathrm{t}_{\mathrm{i}}-\varphi / \theta\right)^{\beta}} \times\left[\mathrm{e}^{-\left(\mathrm{t}_{\mathrm{r}}-\varphi / \theta\right)}\right]^{\mathrm{n}-\mathrm{r}}
\end{aligned}
$$

A função $\log$-likelihood $\mathrm{L}=$ $\ln [\mathrm{L}(\beta ; \theta ; \varphi)]$ será dada por:

$\mathrm{L}=\ln (\mathrm{k})+\mathrm{r} \ln (\beta)-\mathrm{r} \beta \ln (\theta)+(\beta-1) \times$

$\sum_{i=1}^{r} \ln \left(t_{i}-\varphi\right)-\sum_{i=1}^{r}\left(\frac{t_{i}-\varphi}{\theta}\right)^{\beta}-(n-r) \times$

$\left(\frac{\mathrm{t}_{\mathrm{r}}-\varphi}{\theta}\right)^{\beta}$

Para encontrarmos os valores de $\theta$, $\beta$ e $\varphi$ que maximizem a função loglikelihood, obteremos as derivadas de $\theta, \beta$ e de $\varphi$ e as faremos iguais a zero. Então, aplicando alguma álgebra, teremos:

$$
\begin{gathered}
\frac{d L}{d \theta}=-\frac{r \beta}{\theta}+\frac{\beta \times \sum_{i=1}^{r}\left(t_{i}-\varphi\right)^{\beta}}{\theta^{\beta+1}}+ \\
+\frac{\beta(n-r)\left(t_{r}-\varphi\right)^{\beta}}{\theta^{\beta+1}}=0 \\
\frac{d L}{d \beta}=\frac{r}{\beta}-r \ln (\theta)+\sum_{i=1}^{r} \ln \left(t_{i}-\varphi\right)- \\
\sum_{i=1}^{r}\left(\frac{t_{i}-\varphi}{\theta}\right)^{\beta} \times \ln \left(\frac{t_{i}-\varphi}{\theta}\right)-(n-r) \times
\end{gathered}
$$




$$
\begin{gathered}
\left(\frac{\mathrm{t}_{\mathrm{r}}-\varphi}{\theta}\right)^{\beta} \ln \left(\frac{\mathrm{t}_{\mathrm{r}}-\varphi}{\theta}\right)=0 \\
\frac{\mathrm{dL}}{\mathrm{d} \varphi}=-(\beta-1) \sum_{\mathrm{i}=1}^{\mathrm{r}} \frac{1}{\left(\mathrm{t}_{\mathrm{i}}-\varphi\right)}+ \\
\frac{\beta \times\left[\sum_{\mathrm{i}=1}^{\mathrm{r}}\left(\mathrm{t}_{\mathrm{i}}-\varphi\right)^{\beta-1}+(\mathrm{n}-\mathrm{r})\left(\mathrm{t}_{\mathrm{r}}-\varphi\right)^{\beta-1}\right]}{\theta^{\beta}}=0
\end{gathered}
$$

Da equação (5), obteremos:

$$
\theta=\left(\frac{\sum_{i=1}^{r}\left(t_{i}-\varphi\right)^{\beta}+(n-r)\left(t_{r}-\varphi\right)^{\beta}}{r}\right)^{1 / \beta}
$$

Note que quando $\beta=1$, a equação (8) se reduzirá ao estimador "Maximum Likelihood" para a distribuição exponencial de dois parâmetros. Substituindo agora a equação (8) para $\theta$ nas equações (6) e (7) e aplicando alguma álgebra, as equações (6) e (7) se transformam em:

$$
\begin{aligned}
& \frac{r}{\beta}+\sum_{i=1}^{r} \ln \left(t_{i}-\varphi\right)- \\
& r \times\left[\sum_{i=1}^{r}\left(t_{i}-\varphi\right)^{\beta} \ln \left(t_{i}-\varphi\right)+(n-r)\left(t_{r}-\varphi\right)^{\beta} \ln \left(t_{r}-\varphi\right)\right] \\
& \sum_{i=1}^{r}\left(t_{i}-\varphi\right)^{\beta}+(n-r)\left(t_{r}-\varphi\right)^{\beta} \\
& =0 \\
& -\left[\frac{\sum_{\mathrm{i}=1}^{\mathrm{r}}\left(\mathrm{t}_{\mathrm{i}}-\varphi\right)^{\beta}+(\mathrm{n}-\mathrm{r})\left(\mathrm{t}_{\mathrm{r}}-\varphi\right)^{\beta}}{\mathrm{r}}\right] \times \\
& (\beta-1) \sum_{i=1}^{r} \frac{1}{\left(t_{i}-\varphi\right)}+\beta \times \\
& \times\left[\sum_{\mathrm{i}=1}^{\mathrm{r}}\left(\mathrm{t}_{\mathrm{i}}-\varphi\right)^{\beta-1}+(\mathrm{n}-\mathrm{r})\left(\mathrm{t}_{\mathrm{r}}-\varphi\right)^{\beta-1}\right]=0(10)
\end{aligned}
$$

O problema se reduziu ao de se obter uma solução simultânea para as duas equações interativas (9) e (10). A solução simultânea de duas equações interativas pode parecer um problema relativamente simples, quando comparado com a árdua tarefa de se resolver simultaneamente três equações interativas, como descrito por Harter et al., (1965).

O método de estimação padrão do "Maximum Likelihood" quando utilizado na estimação dos três parâmetros do modelo Weibull poderá apresentar problemas, devido ao fato de que as condições de regularidade não serem obtidas, veja Murthy et al., (2004), Blischke, (1974) e Zanakis and Kyparisis, (1986). Pesquisadores freqüentemente têm discutido a existência de soluções para o sistema anterior formado pelas equações (9) e (10), devido poder-se ter mais de uma solução para esse sistema, ou mesmo, nenhuma solução possível; veja Zanakis and Kyparisis (1986). Para se resolver esse problema de falta de regularidade, um dos métodos propostos por Cohen, et al. (1984) é o de se substituir a equação (10) pela equação:

$$
\mathrm{E}(\varphi)=\varphi=\mathrm{t}_{1}-\frac{\theta}{\mathrm{n} / \beta} \Gamma\left(\frac{1}{\beta}+1\right)
$$

Aqui, $t_{1}$ representa a primeira ordem estatística de uma amostra de tamanho n. O Anexo (2) apresenta a solução da equação (11). Com $\theta$ dado pela equação (8), finalmente teremos:

$$
\begin{gathered}
\mathrm{t}_{1}-\left(\frac{\sum_{\mathrm{i}=1}^{\mathrm{r}}\left(\mathrm{t}_{\mathrm{i}}-\varphi\right)^{\beta}+(\mathrm{n}-\mathrm{r})\left(\mathrm{t}_{\mathrm{r}}-\varphi\right)^{\beta}}{\mathrm{r} \times \mathrm{n}}\right)^{1 / \beta} \times \\
\times \Gamma\left(\frac{1}{\beta}+1\right)=0
\end{gathered}
$$

$\mathrm{Na}$ solução das equações do estimador "Maximum Likelihood", iremos utilizar essa sugestão proposta por Cohen et al., (1984). Desse modo, o problema foi reduzido à solução simultânea das duas equações interativas (9) e (12).

\section{EXEMPLO}

Esse exemplo está relacionado com um novo componente metalúrgico sendo submetido a um teste de vida. 
Como esse é um novo produto, um novo tipo de aço de alta resistência e baixa liga, no qual o componente vanádio foi substituído pelo nióbio, existe muito pouca informação disponível acerca dos possíveis valores que os três parâmetros da distribuição de amostragem Weibull possam ter.

Um teste de vida preliminar é realizado para se determinar um valor estimado para os parâmetros da distribuição de amostragem. Nesse teste preliminar, um grupo de doze vigas foi submetido a um teste de vida, com todas as vigas testadas (cicladas) até a ocorrência da nona falha. A Tabela (1) seguinte apresenta os tempos de falhas (ciclos) para esse teste de vida preliminar.

Tabela1. Tempos de falhas (ciclos) para o teste de vida preliminar.

\begin{tabular}{|c|c|c|}
\hline 3.322 .329 & 4.023 .048 & 2.934 .330 \\
\hline 3.781 .710 & 2.780 .470 & 2.251 .930$. \\
\hline 4.517 .904 & 3.154 .093 & 3.568 .961 \\
\hline
\end{tabular}

A distribuição de amostragem é o modelo Weibull de três Parâmetros. Utilizando-se o estimador "Maximum Likelihood" para o parâmetro de forma $\beta$, para o parâmetro de escala $\theta$ e para o parâmetro de vida mínima $\varphi$ do modelo Weibull para um teste de vida truncado por falhas, obteremos os seguintes valores para esses três parâmetros: $\beta=$ 3,$24 ; \theta=3.592 .409$ ciclos; $\varphi=555,600$ ciclos.

Um teste de vida seqüencial foi então desenvolvido para se avaliar os valores estimados obtidos para esses parâmetros do modelo Weibull de amostragem. Foi decidido que o valor de $\alpha$ seria de 0,05 e o valor de $\gamma$ seria de 0,10 . Escolheram-se os seguintes valores para os parâmetros da hipótese nula $\mathrm{H}_{0} \mathrm{e}$ da hipótese alternativa $\mathrm{H}_{1}$ : parâmetro de escala alternativo $\theta_{1}=3.000 .000$ ciclos, parâmetro de forma alternativo $\beta_{1}=3,0$, parâmetro de vida mínima alternativo $\varphi_{1}$ $=500.000$ ciclos, parâmetro de escala nulo $\theta_{0}=3.592 .409$ ciclos, parâmetro de forma nulo $\beta_{0}=3,24$, parâmetro de vida mínima nulo $\varphi_{0}=555.600$ ciclos. Nesse exemplo, mesmo após a análise de 15 itens ou observações, não foi possível obter-se uma decisão de se aceitar ou de se rejeitar a hipótese nula $\mathrm{H}_{0}$. Ao tempos de falhas (ciclos) obtidos foram os seguintes:

$\begin{array}{lll}2.495 .133 ; & 3.937 .873 ; & 2.169 .998 \\ 2.643 .848 ; & 4.056 .386 ; & 3.178 .130 \\ 2.615 .244 ; & 4.867 .873 ; & 5.093 .244 ; \\ 3.081 .700 ; & 4.969 .168 ; & 2.430 .828 \\ 2.029 .255 ; & 2.556 .433 ; & 4.023 .048\end{array}$

O resultado do teste de vida seqüencial para o modelo Weibull de três parâmetros é o apresentado na Figura 1.

Resolvendo-se agora as equações (3) e (4), com os valores de $\theta_{1}=$ 3.000.000 ciclos; $\theta=\theta_{0}=3.592 .409$ ciclos; $\varphi=\varphi_{0}=555.600$ ciclos; $\varphi_{1}=$ 500.000 ciclos; $\beta_{1}=3,0 ; \beta=\beta_{0}=3,24 ; \alpha$ $=0,05 ; \gamma=0,10$ e fazendo $\mathrm{P}(\theta, \beta, \varphi)$ ser igual a 0,01 , poderemos então calcular o tamanho esperado da amostra para efeito de truncagem $E(n)$ desse teste de vida seqüencial sendo analisado. Desse modo, teremos:

$$
\begin{gathered}
\mathrm{E}(\mathrm{n}) \approx \frac{\mathrm{P}(\theta, \beta) \ln \mathrm{A}+[1-\mathrm{P}(\theta, \beta)] \ln \mathrm{B}}{\mathrm{E}(\mathrm{w})} \\
\mathrm{E}(\mathrm{n}) \approx \frac{2,8840}{0,2911} ; \mathrm{E}(\mathrm{n}) \approx 9,907 \approx 10 \text { itens }
\end{gathered}
$$

Logo, poderemos tomar uma decisão a respeito de se aceitar ou se rejeitar a hipótese nula $\mathrm{H}_{0}$ após a análise da observação de número 10 . 


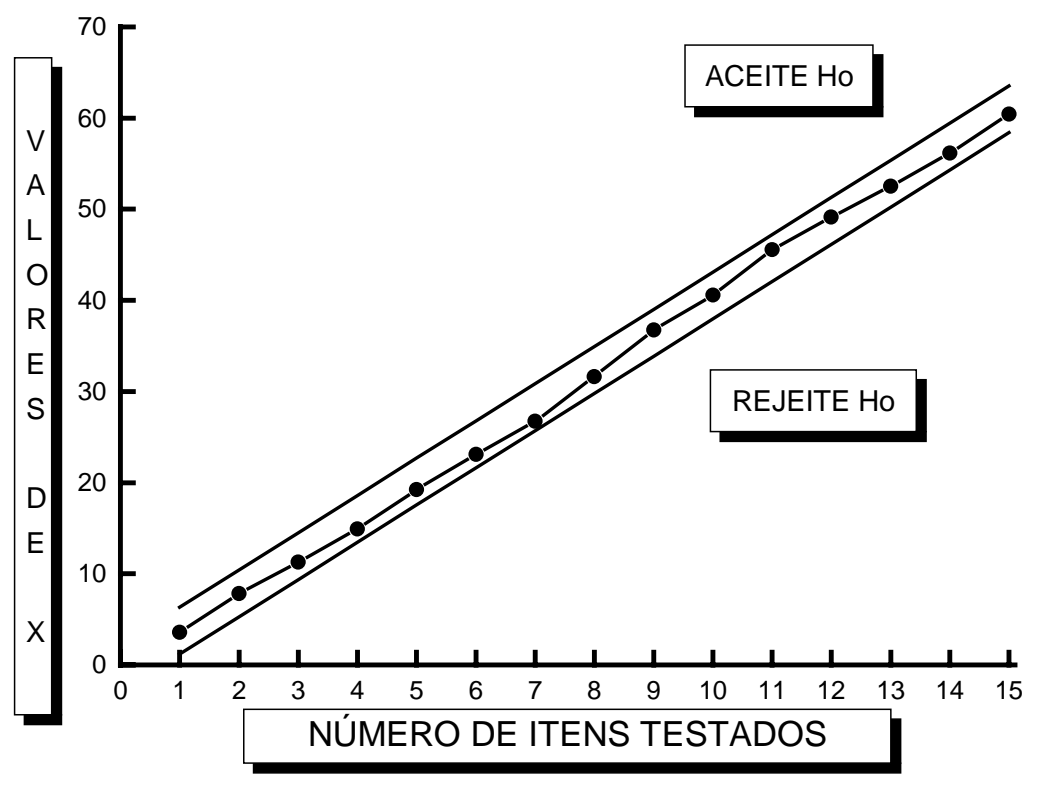

Figura 1. Resultados do teste de vida seqüencial para o modelo Weibull de três parâmetros.

6. UM PROCEDIMENTO PARA SE APLICAR O MECANISMO DE TRUNCAGEM

De acordo com Kapur e Lamberson (1977), quando o ponto de truncagem é alcançado, é traçada uma linha dividindo ao meio o gráfico seqüencial, como mostrado na Figura (2) seguinte. Essa linha é traçada iniciandose na origem do gráfico, paralela às linhas de aceitação e de rejeição. A decisão de se aceitar ou se rejeitar a hipótese nula H0 irá depender simplesmente de que lado a observação final se encontra.

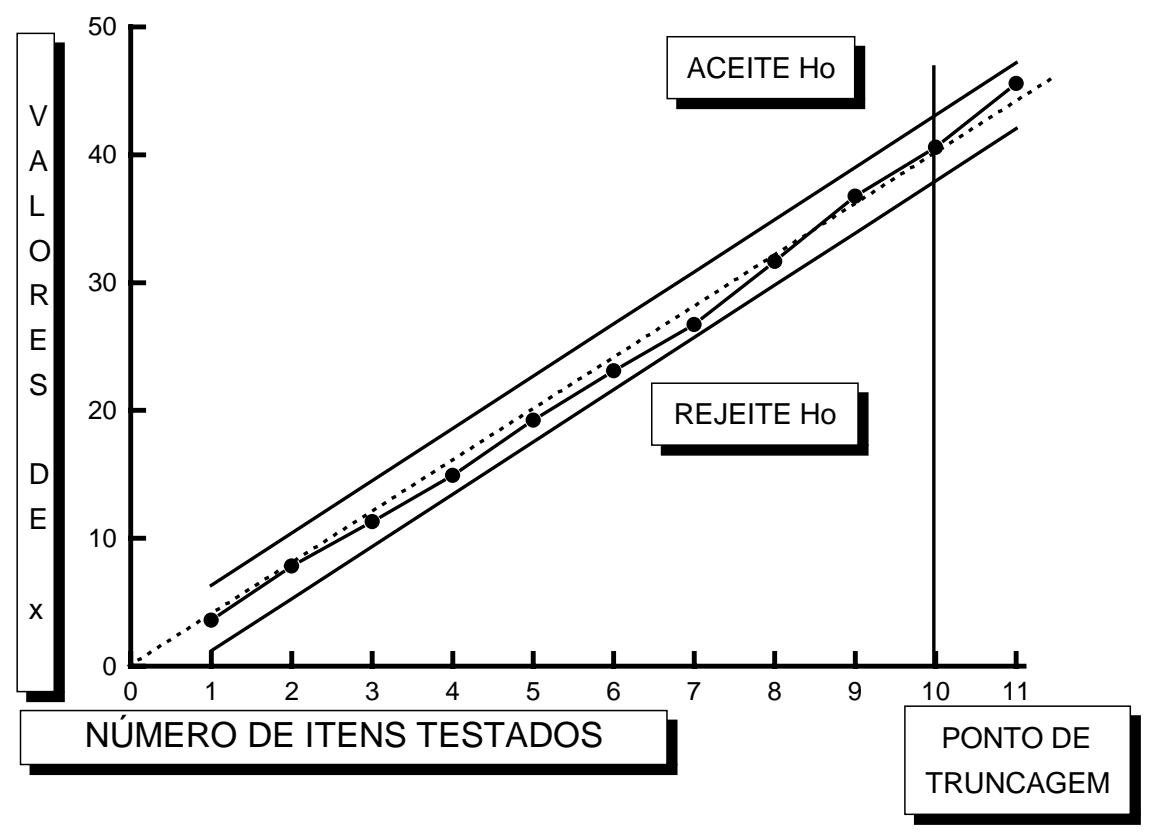

Figura 2. Um procedimento para se aplicar o mecanismo de truncagem.

Obviamente esse procedimento de truncagem altera os níveis ou valores de ENGEVISTA, v. 8, n. 2, p. 110-119, dezembro 2006 $\alpha$ e de $\gamma$ do teste de vida original. Entretanto, de acordo com Kapur e 
Lamberson (1977), essa mudança será muito pequena se o ponto de truncagem não for muito pequeno (menor do que três unidades). Como podemos observar na Figura (2), a hipótese nula $\mathrm{H}_{0}$ deverá ser aceita, pois a observação final (observação de número 10), se encontra no lado da linha relacionado com a aceitação de $\mathrm{H}_{0}$.

\section{CONCLUSÕES}

Nesse trabalho, desenvolvemos um teste de vida seqüencial no qual a distribuição de amostragem é o modelo Weibull de três parâmetros, e o produto sendo analisado é um componente metalúrgico que foi recentemente modificado. Desse modo, existe muito pouca informação disponível sobre os valores que os três parâmetros desse modelo Weibull poderiam ter. $\mathrm{O}$ parâmetro de localização ou de vida mínima foi considerado ser diferente de zero. Para estimarmos os parâmetros de forma, escala e de vida mínima do modelo Weibull de três parâmetros, utilizamos o estimador "Maximum Likelihood" em uma situação de teste de vida truncado por falhas. Como vimos anteriormente, o método de estimação padrão do "Maximum Likelihood" quando utilizado na estimação dos três parâmetros do modelo Weibull poderá apresentar problemas, devido ao fato de que as condições de regularidade não serem obtidas, veja Murthy et al., (2004), Blischke, (1974) e Zanakis and Kyparisis, (1986). Para resolvermos esse problema de falta de "regularidade" ou "aderência", utilizamos uma modificação proposta por Cohen, et al. (1984). Desenvolvemos também um mecanismo de truncagem para a distribuição de amostragem Weibull de três parâmetros. $\mathrm{Na}$ determinação de uma expressão para o tamanho esperado da amostra E(n) para efeito de truncagem de um teste de vida seqüencial, aplicamos ao modelo Weibull de três parâmetros um mecanismo desenvolvido por Mood and Graybill (1963) para a distribuição normal. Fornecemos regras para truncarmos nosso teste de vida seqüencial tomando uma das ENGEVISTA, v. 8, n. 2, p. 110-119, dezembro 2006 duas decisões possíveis no momento da truncagem, ou seja; aceitarmos a hipótese nula $\mathrm{H}_{0}$ ou rejeitarmos a hipótese nula $\mathrm{H}_{0}$. Para determinarmos o tamanho esperado da amostra $E(n)$ do teste de vida seqüencial para efeito de truncagem, tivemos de utilizar um processo de integração numérica (utilizamos nesse trabalho a regra de Simpson 1/3). Sem a utilização do mecanismo de truncagem desenvolvido nesse trabalho, não fomos capazes de obter uma decisão acerca de aceitarmos ou rejeitarmos a hipótese nula $\mathrm{H}_{0}$, mesmo após termos analisado 15 observações. Aplicando agora o mecanismo de truncagem desenvolvido, a decisão de aceitarmos a hipótese nula foi alcançada com a análise de apenas 10 observações ou itens. Esse fato mostra a vantagem de se utilizar esse tipo de mecanismo de truncagem em um teste de vida seqüencial.

\section{BIBLIOGRAFIA}

BAIN, Lee J. (1978). "Statistical Analysis of Reliability and Life-Testing Models. Theory and Methods", Marcel Dekker, Inc., New York, 1978.

BLISCHKE, W. R. (1974). On nonregular estimation. II. Estimation of the Location Parameter of the Gamma and Weibull Distributions, "Communications in Statistics", 1974, Vol. 3, pp. 1109-1129.

COHEN, A. C.; WHITTEN, B. J. and DING, Y. (1984). Modified Moment Estimation for the Three-Parameter Weibull Distribution. "Journal of Quality Technology", 1984, Number 16, pp.159167.

DE SOUZA, Daniel I. (2004a). Application of a Sequential Life Testing with a Truncation Mechanism for an Underlying Three-Parameter Weibull Model. "ESREL 2004-PSAM 7 Conference", edited by Spitzer, Schmoker and Dang, Springler-Verlang, Germany, Berlin, 2004a, Vol. 3, pp. 1674-1680.

DE SOUZA, Daniel I. (2004b). Sequential Life Testing Approach 
Applied to Metallurgical Products when the Weibull Model Minimum Life is Different from Zero. "CONAMET/SAM 2004", edited and published by the University of La Serena, Chile, La Serena, pp. 445-450.

DE SOUZA, Daniel I. (2003). Sequential life testing with a truncation mechanism for an underlying Three-parameter Weibull model, ICheap-6, Chemical Engineering Transactions, Vol. 1 p. 557562, Sauro Pierucci (ed), Pisa, Italy.

DE SOUZA, Daniel I. (2001). Sequential life testing with a truncation mechanism for an underlying Weibull model, Towards a Safer World, ESREL 2001 Conference, Zio, Demichela \& Piccinini (eds), Torino, Italy, 16 - 20 September 2001; 3: 1539 - 1546, Politecnico Di Torino.

HARTER, H. et al. (1965). Maximum Likelihood Estimation of the Parameters of Gamma and Weibull Populations from Complete and from Censored Samples.
“Technometrics", 1965, Number 7, pp. 639-643; erratum, 15 (1973), pp. 431.

KAPUR, Kailash \& Lamberson, LEONARD R. (1997). Reliability in Engineering Design. New York: John Willey \& Sons, Inc.

MOOD, A. M. and GRAYBILL, F. A. (1963). "Introduction to the Theory of Statistics", Second Edition, McGrawHill, New York, 1963.

MURTHY, D. N. P; XIE, M. and HANG, R. (2004). "Weibull Models" Wiley Series in Probability and Statistics, John Wiley \& Sons, Inc., New Jersey, 2004.

ZANAKIS, S. H. and KYPARISIS, J. (1986). A Review of Maximum Likelihood Estimation Methods for the Three Parameter Weibull Distribution. "Journal of Statistical Computation and Simulation”, 1986, Number 25, pp. 5373.

\section{ANEXO 1. SOLUÇÃO DOS COMPONENTES DA EQUAÇÃO (4)}

$$
\mathrm{E}\left[\left(\mathrm{t}-\varphi_{1}\right)^{\beta_{1}}\right]=\int_{\phi_{1}}^{\infty}\left(\mathrm{t}-\varphi_{1}\right)^{\beta_{1}} \frac{\beta}{\theta}\left(\frac{\mathrm{t}-\varphi_{1}}{\theta}\right)^{\beta-1} \exp \left[-\left(\frac{\mathrm{t}-\varphi_{1}}{\theta}\right)^{\beta}\right] \mathrm{dt}
$$

Fazendo $U=\left(\frac{\mathrm{t}-\varphi_{1}}{\theta}\right)^{\beta}$, teremos $\mathrm{du}=\frac{\beta}{\theta}\left(\frac{\mathrm{t}-\varphi_{1}}{\theta}\right)^{\beta-1} \mathrm{dt} ; \quad \mathrm{t}=\varphi_{1}+\theta \mathrm{U}^{1 / \beta}$.

Quando $t \rightarrow \infty, U \rightarrow \infty$. Agora, quando $t \rightarrow \varphi, \quad U \rightarrow 0$. Logo, a Equação (A1) se transformará em:

$\mathrm{E}\left[\left(\mathrm{t}-\varphi_{1}\right)^{\beta_{1}}\right]=\theta^{\beta_{1}} \int_{0}^{\infty} \mathrm{U}^{\beta_{1} / \beta} \mathrm{e}^{-\mathrm{U}} \mathrm{du}$. Essa integral é a função gama $\Gamma\left(1+\frac{\beta_{1}}{\beta}\right)$. Logo, teremos:

$$
\mathrm{E}\left[\left(\mathrm{t}-\varphi_{1}\right)^{\beta_{1}}\right]=\theta^{\beta_{1}} \Gamma\left(1+\frac{\beta_{1}}{\beta}\right)
$$

Utilizando-se as mesmas transformações empregadas na solução da Equação (A1), teremos:

$$
\mathrm{E}\left[\left(\mathrm{t}-\varphi_{0}\right)^{\beta_{0}}\right]=\theta^{\beta_{0}} \Gamma\left(1+\frac{\beta_{0}}{\beta}\right)
$$


No cálculo do $E\left[\ln \left(t-\varphi_{1}\right)\right]$, teremos:

$$
\mathrm{E}\left[\ln \left(\mathrm{t}-\varphi_{1}\right)\right]=\int_{\phi_{1}}^{\infty} \ln \left(\mathrm{t}-\varphi_{1}\right) \frac{\beta}{\theta}\left(\frac{\mathrm{t}-\varphi_{1}}{\theta}\right)^{\beta-1} \exp \left[-\left(\frac{\mathrm{t}-\varphi_{1}}{\theta}\right)^{\beta}\right] \mathrm{dt}
$$

Fazendo novamente $U=\left(\frac{\mathrm{t}-\varphi_{1}}{\theta}\right)^{\beta}$, a equação (A4) se transformará em:

$$
E\left[\ln \left(t-\varphi_{1}\right)\right]=\int_{0}^{\infty} \ln \left(\theta \mathrm{U}^{1 / \beta}\right) \mathrm{e}^{-\mathrm{U}} \mathrm{du}=\ln (\theta) \int_{0}^{\infty} \mathrm{e}^{-\mathrm{U}} \mathrm{du}+\frac{1}{\beta} \int_{0}^{\infty} \ln (\mathrm{U}) \mathrm{e}^{-\mathrm{U}} \mathrm{du} . \text { Logo: }
$$

$$
\mathrm{E}\left[\ln \left(\mathrm{t}-\varphi_{1}\right)\right]=\ln (\theta)+\frac{1}{\beta} \int_{0}^{\infty} \ln (\mathrm{U}) \mathrm{e}^{-\mathrm{U}} \mathrm{du}
$$

A integral acima tem de ser resolvida através do emprego de algum processo de integração numérica, como por exemplo, a Regra de Simpson 1/3. Como recordamos, a regra de Simpson $1 / 3$ é dada por:

$$
\begin{aligned}
& \int_{\mathrm{a}}^{\mathrm{b}} \mathrm{f}(\mathrm{x}) \mathrm{dx}=\frac{\mathrm{g}}{3}\left(\mathrm{f}_{1}+4 \mathrm{f}_{2}+2 \mathrm{f}_{3}+\cdots+4 \mathrm{f}_{\mathrm{n}}+\mathrm{f}_{\mathrm{n}+1}\right)-\text { erro. Fazendo-se o erro }=0, \text { obteremos: } \\
& \int_{0}^{\infty} \ln (\mathrm{U}) \mathrm{e}^{-\mathrm{U}} \mathrm{du}=\frac{\mathrm{g}}{3} \times\left(\ln \left(\mathrm{U}_{1}\right) \mathrm{e}^{-\mathrm{U}_{1}}+4 \ln \left(\mathrm{U}_{2}\right) \mathrm{e}^{-\mathrm{U}_{2}}+\cdots+\ln \left(\mathrm{U}_{\mathrm{n}+1}\right) \mathrm{e}^{-\mathrm{U}_{\mathrm{n}+1}}\right) . \text { Logo, }
\end{aligned}
$$

teremos:

$$
\begin{gathered}
\mathrm{E}\left[\ln \left(\mathrm{t}-\varphi_{1}\right)\right]=\ln (\theta)+\frac{1}{\beta} \times \frac{\mathrm{g}}{3} \times\left\{\sum_{\mathrm{i}=1}^{\mathrm{n}+1}\left[\ln \left(\mathrm{U}_{\mathrm{i}}\right) \mathrm{e}^{-\mathrm{U}_{\mathrm{i}}} \times(1,2 \text { or } 4)\right]\right\} ; \mathrm{i}=1,2, \ldots \mathrm{n}+1 \\
\mathrm{U}=\left(\frac{\mathrm{t}-\phi_{1}}{\theta}\right)^{\beta}
\end{gathered}
$$

Utilizando-se as mesmas transformações empregadas na solução da Equação (A4), obteremos:

$$
\mathrm{E}\left[\ln \left(\mathrm{t}-\varphi_{0}\right)\right]=\ln (\theta)+\frac{1}{\beta} \times \frac{\mathrm{g}}{3} \times\left\{\sum_{\mathrm{i}=1}^{\mathrm{n}+1}\left[\ln \left(\mathrm{Y}_{\mathrm{i}}\right) \mathrm{e}^{-\mathrm{Y}_{\mathrm{i}}} \times(1,2 \text { or } 4)\right]\right\} ; \mathrm{Y}=\left(\frac{\mathrm{t}-\phi_{0}}{\theta}\right)^{\beta}
$$

\section{ANEXO 2. DETERMINANDO-SE UM ESTIMADOR INICIAL PARA A VIDA MÍNIMA $\varphi$}

A função densidade $(p d f)$ de $\mathrm{t}_{1}$ será dada por: $\mathrm{f}\left(\mathrm{t}_{1}\right)=\mathrm{n}\left[1-\mathrm{F}\left(\mathrm{t}_{1}\right)\right]^{\mathrm{n}-1} \mathrm{f}\left(\mathrm{t}_{1}\right)$.

Agora, como $F\left(t_{1}\right)=1-R\left(t_{1}\right)$, obteremos: $f\left(t_{1}\right)=n\left[R\left(t_{1}\right)\right]^{n-1} f\left(t_{1}\right)$ 
Para a distribuição de amostragem Weibull de três parâmetros, teremos:

$$
\mathrm{f}\left(\mathrm{t}_{1}\right)=\frac{\mathrm{n} \beta}{\theta}\left(\frac{\mathrm{t}-\varphi}{\theta}\right)^{\beta-1}\left\{\exp -\left[\left(\frac{\mathrm{t}-\varphi}{\theta}\right)^{\beta}\right]\right\}^{\mathrm{n}}
$$

O valor esperado de $\mathrm{t}_{1}$ será dado por: $\mathrm{E}\left(\mathrm{t}_{1}\right)=\int_{\varphi}^{\infty} \frac{\mathrm{n} \beta}{\theta} \mathrm{t}\left(\frac{\mathrm{t}-\varphi}{\theta}\right)^{\beta-1}\left\{\exp -\left[\left(\frac{\mathrm{t}-\varphi}{\theta}\right)^{\beta}\right]\right\}^{\mathrm{n}} \mathrm{dt}$

Fazendo-se $\mathrm{U}=\left(\frac{\mathrm{t}-\varphi}{\theta}\right)^{\beta} ; \mathrm{du}=\frac{\beta}{\theta}\left(\frac{\mathrm{t}-\varphi}{\theta}\right)^{\beta-1} \mathrm{dt} ; \mathrm{dt}=\frac{\mathrm{du}}{\frac{\beta}{\theta}\left(\frac{\mathrm{t}-\varphi}{\theta}\right)^{\beta-1}} ; \mathrm{t}=\theta \mathrm{U}^{1 / \beta}+\varphi$

Quando $t \rightarrow \infty$; $\mathrm{U} \rightarrow \infty$; Agora, quando $t \rightarrow \varphi ; \mathrm{U} \rightarrow 0$. Logo:

$$
\begin{gathered}
E\left(t_{1}\right)=\int_{0}^{\infty} n\left(\theta U^{1 / \beta}+\varphi\right) e^{-n U} d u=\theta \int_{0}^{\infty} n U^{1 / \beta} e^{-n U} d u+\varphi \int_{0}^{\infty} n e^{-n U} d u, \text { onde } \\
\varphi \int_{0}^{\infty} n e^{-n U} d u=-\varphi\left[e^{-n U}\right]_{0}^{\infty}=-\varphi[0-1]=\varphi
\end{gathered}
$$

Na solução da integral $\theta \int_{0}^{\infty} n U^{1 / \beta} e^{-n U} d u$, faça $Z=n U ; d u=\frac{d z}{n} ; U=\frac{Z}{n}$.

Quando $\mathrm{U} \rightarrow \infty ; \mathrm{Z} \rightarrow \infty$; Quando $\mathrm{U} \rightarrow 0 ; \mathrm{Z} \rightarrow 0$. Então:

$$
E\left(t_{1}\right)=\theta \int_{0}^{\infty}\left(\frac{Z}{n}\right)^{1 / \beta} e^{-z} d z+\varphi=\frac{\theta}{n^{1 / \beta}} \int_{0}^{\infty} z^{1 / \beta} e^{-z} d z+\varphi
$$

Resolvendo-se essa integral, obteremos: $E\left(t_{1}\right)=t_{1}=\frac{\theta}{n^{1 / \beta}} \Gamma\left(\frac{1}{\beta}+1\right)+\varphi$. Vemos finalmente então que $\varphi$ poderá ser então estimada por:

$$
\mathrm{E}(\varphi)=\varphi=\mathrm{t}_{1}-\frac{\theta}{\mathrm{n}^{1 / \beta}} \Gamma\left(\frac{1}{\beta}+1\right)
$$

